\title{
TENTATIVA DE VERIFICAÇÃO DA SENSIBILIDADE DE UM INSTRUMENTO PARA AVALIAR ASPECTOS BIOLOGICOS DOS CUIDADOS DE ENFERMAGEM
}

* Eloita P. Neves

* Lindalva L. Ricker

* Dalva dos Reis Trindade

* Maria Albertina B. Santos

* Maria Augusta R. Santos

* Sonia Maria A. Moraes

RBEn/05

NEVES, E.P. e colaboradores - Tentativa de verificaçāo da sensibilidade de um instrumento para avaliar aspectos biológicos dos cuidados de enfermagem. Rev. Bras. En?.; DF, $29: 42-52,1976$.

\section{INTRODUÇAO:}

Nos Estados Unidos, conforme refere Zimmer (1974), o Social Security solicitou aos profissionais da área da saúde que organizassem e implementassem sistemas e métodos de controle efetivo da qualidade de seus serviços profissionais até o ano de 1976. Por este motivo, entre outros, as enfermeiras norte americanas têm incrementado estudos com a finalidade de encontrar critérios de avaliação que permitam atender a essa solicitação.

Não se tem conhecimento, até o momento, da existência em nosso país de exigências sobre controle da qualidade da assistência prestada, bem como não foram ainda estabelecidos meios objetivos que possibilitem identificar o nível dos cuidados recebidos pelos pacientes.

Seria interessante que as(os) enfermeiras(os) brasileiras (os), independente de solicitação oficial refletissem sobre a importância do assunto. Não se pode mais seguir exclusivamente os manuais de rotinas e as ordens médicas. Urge que se acompanhe o rítimo de mudança que se processa no mundo moderno. Como disse Abdellah, citada por Zimmer (1974) "no mundo moderno, a adaptação à mudança é essencial para a sobrevivência e a inovação uma condição para o sucesso". Assegurar assistência de enfermagem da melhor qualidade segundo Zimmer (1974), pode ser vista como uma

- Mestrandas do ano 1976 - Escola de Enfermagem da UFRJ. 
NEVES, E.P. e colaboradores - Tentativa de verificação da sensibilidade de um instrumento para avaliar aspectos biológicos dos cuidados de enfermagem. Rev. Bras. Enf.; DF, 29 : 42-52, 1976.

mudança planejada e requerida para a sobrevivência da profissão e uma condição para o seu sucesso. Para garantir a qualidade é preciso avaliar continuadamente a assistência de enfermagem prestada.

Bloch (1975) inclui como fatores que devem ser considerados na avaliação da assistência: a estrutura, o processo e os resultados desta assistência sobre o paciente.

Pesquisas preliminares sobre avaliação, enfatizavam a estrutura com seus recursos materiais e organização dos serviços. Posteriormente, passou-se a dar importância aos estudos de atividades das enfermeiras. Todos estes trabalhos foram valiosos, pois indicaram uma direção para a enfermagem como profissão, mas, não mediram os efeitos dos cuidados sobre o paciente, o que nos Estados Unidos passou a ser feito recente.

Por outro lado, é possível que a excelência dos recursos da estrutura e a excelência do desempenho dos profissionais da saúde, influenciem na obtenção de bons resultados sobre as condições de saúde e bem estar dos pacientes.

Taylor (1974) em seus estudos levantou três suposições:

“1) A assistência de enfermagem afeta o resultado da doença sobre o paciente e influi nas suas futuras condições de saúde;

2) embora a enfermagem divida as responsabilidades da assistência ao paciente com outras disciplinas da área da saúde, há resultados que são originalmente atribuídos à assistência de enfermagem e que podem ser identificados;

3) há resultados que são parcialmente ou marginalmente atribuíveis à assistência de enfermagem e que podem também ser identificados".

Mesmo dividindo a assistência ao paciente com outros profissionais da saú- de, é de se reconhecer que a(0) enfermeira (o) seja responsável pelo resultado final dessa assistência, pela garantia de sua qualidade.

A garantia da qualidade pode, entre outros meios, ser conseguida através da implementação da avaliação sistemática e da ação levada à efeito visando melhorar constantemente essa qualidade.

Para valiar é preciso partir da determinaçāo de critérios específicos, que infelizmente ainda não foram estabelecidos, em nosso país. A maior dificuldade em desenvolver esses critérios parece relacionar-se com os escassos estudos feitos a esse respeito, e com a inexistência de padrões de assistência de enfermagem.

Abdellah (1961) já se preocupava com o assunto, salientando que as dificuldades em identificar critérios têm levado muitas investigações para áreas onde há maiores facilidades. Um dos fatores que contribui para essa dificuldade é a existência de muitas variáveis no ambiente e no paciente.

Zimmer e colaboradoras (1974), no Simpósio sobre Guidelines for Development of Outcome Criteria, sugerem orientações básicas que facilitam o desenvolvimento dos trabalhos de avaliação. Apresentam 29 itens, dos quais ressalta-se que os critérios devem ser pertinentes a um quadro de referência que pode ser baseado em recursos, atividades ou resultados. Os critérios de resultado sempre se relacionam com objetivos estabelecidos e com as necessidades de uma população específica de pacientes, em determinado estágio do ciclo saúde-enfermidade. Deve ser testado o quadro de referência para verificar sua praticabilidade e aplicabilidade, compreensão e aceitação por parte das(os) enfermeiras(os) que trabalham com determinada população específica. 
NEVaS, F.P. e colaboradores - Tentativa de verificaçăo da sensibilidade de um instrumento para avaliar aspectos biologicos dos culdados de enfermagem. Rev. Bras. Enf.; DF, 29 : 42-52, 1976.

Taylor (1974) em seu relatórío prellminar do estudo para estabelecer critérios de resultados da assistência de enfermagem a pacientes neurológicos hospitalizados, apresenta três modelos para avaliação correspondendo ao período crítico, a fase que val do final do período crítico até a alta hospitalar, e a fase correspondente aos dols meses após a alta.

Brownle et Collins (1974') testaram a utilização de um instrumento para medir a assistência de enfermagem relaclonada com as necessidades fisiológicas de pacientes com acidente vascular cerebral, na fase aguda da doença. Apesar do limitado número de casos observados, concluiram ter o instrumento sensibilidade para estabelecer as diferenças de níveis dos cuidados de enfermagem que estavam sendo ministrados aos pacientes selecionados para o estudo. As mesmas autoras afirmaram que 0 instrumento poderia servir não somente para avaliação, como também poderia ser to mado como guia de cuidados, no aspecto físiológico, naquela fase da doença.

$\mathrm{Na}$ presente pesquisa foram focalizados apenas os resultados dos cuidados de enfermagem prestados aos pacientes com acldente vascular cerebral após 72 horas da Instalação da doença até a 4.a semana de Internação, em três hospitais oflciais com mais de 300 leitos, sem considerar a eficiência destas intervençōes.

Partiu-se da hipótese de que serla passível medir a qualidade dos cuidados prestados aos pacientes com A.V.C. mediante a utilização de um instrumento onde constavam itens relacionados com os cuidados básicos ideais e itens relaclonados com as anotaçōes de enfermagem nos prontuários. Berg (1974) considera essencial a documentação dos cuidados proporcionados: "sem documentação não há "input" de enfermagem no sistema de informação de saúde e os re- sultados das intervençōes de enfermagem não podem ser valldados".

Cada paciente fol visitado no periodo da manhã e no período noturno por três enfermeiras que, simultaneamente, utillzando cada uma um formulário e evitando comunicar-se entre si, observaram e registraram as ocorrências. Kaplan (1972) afirma que para reduzir os erros de medida é de particular importância a pessoa do observador e a sensibilidade do instrumento utilizado. A medida inclui dois componentes: um que corresponde à extensāo ou grandeza a medir, e outro que corresponde a "outros fatores difícels de controlar". Também diz que uma medida é válida quando mede aquilo que se propōe medir, mas que ela pode não estar livre de erro. "O erro é, em si, medida de nosso insucesso no sentido de alcançar o que pretendiamos; a validez diz respeito à significação clentifica de nossas aspiraçōes. Nenhuma aspiração humana se concretiza completamente; nenhuma medida está inteiramente livre de erro. Não obstante, como seres humanos, podemos esperar razoavelmente - e, como clentistas, esforçarnos dedicadamente - para reduzir o erro ao mínimo".

Fol utllizado, pelas pesquisadoras, como critério de medida de qualidade, 0 atendimento a determinados aspectos relaclonados com as necessidades blológicas dos pacientes, que constitulam os problemas de enfermagem listados no instrumento. Du Gaz (1974) reconhece na teoria das necessidades humanas de Maslow, uma base útil para estabelecer prioridades nas intervençöes de enfermagem. Essas intervençōes incluem 0 atendimento às necessidades emocionais, sociais, espirituais e fisiológicas. Da mesma forma se manifestaram Brownie et Collins (1974) Justificando o motivo da escolha das necessidades fisiológicas como parâmetro de seu estudo: “... somente depois que as necessidades fisio- 
NEVWS, E.P. e colaboradores - Tentativa de verificaca da sensiblildade de um instrumento pars avaliar aspectos blológicos dos culdados de enfermagem. Rev. Bres Enf.; DF, 29 : 42-52, 1976.

logfcas forem satisfeltas, as outras emergirăo completamente". Os aspectos relaclonados com as necessdades emocionals e psicossocials, constituem desaflo para estudos futuros.

Conforme poderá ser constatado na discussão dos dados flcou comprovada, através dos estudos de Inferência estatistica, a sensibilidade do instrumento utllizedo e foram sugeridos alguns pontos para o aprofundamento de estudos.

\section{METODOLOGIA:}

Para elaboração desta fase, segulramse os passos sugeridos por Joyce Taylor (1974).

\section{DEFINICGAOO DE POPULAÇAO:}

1.1. Critérios para a escolha dos pacientes:

Foram escolhidos paclentes com diagnóstico médico de Acidente Vascular Cerebral, após 72 horas de internaçāo na clínica até a 4.a semans, independente de seso, Idade, nível de consclêncla, condiçōes de comunicação e/ou de deambulação.

\subsection{Determinação de amostra:}

No 1.0 teste, entre a populaçāo de 17 pacientes selecionados, foram utilizados 16, assim distribuídos: 4 no hospital I, 4 no hospital II e 8 no hospital III.
1.3. Critérios para a escolha do Hospital:

- ser oficlal;

- passuir no minimo 1 enfermelra em cada turno na clínlca em estudo;

- aceltação por parte da administraçăo e da equipe de enfermagem;

- aceltação por parte dos pacientes consclentes.

\section{DSQUEMA DE RHFHRENCLA:}

Com base na metodologla utlizada por Brownie et Collins (1974), na qual 3 pares de avaliadoras visitavam os paclentes slmultaneamente na 5.a e 72.a hora após a admissão, o grupo de pesquisadoras estabeleceu um método que será descrito no item 7.

\section{IDENTIFICAÇAO DOS PROBLPMAS COMUNS AO GRUPO DE PACIEN- TES SELECIONADOS:}

A partír da revisão da literatura e da experiêncla pessoal das pesquisadoras, foram Identificados e agrupados os problemas básicos de enfermagem relaclonados com aspectos blológicos e elaboradas perguntas em formulárlo especifico.

\section{ESTABELECDMENTO DOS CRITE- RIOS DE MENSURAÇA:}

\subsection{Niveis de cuidados:}

Para avaliar os resultados foram estabelecidos 4 nívels de culdados de enfermagem:

$$
\begin{aligned}
& \text { Nível I - } 90 \text { a } 100 \% \text { = alta qualidade; } \\
& \text { Nível II - } 50 \text { a } 89 \% \text { = moderada qualidade; } \\
& \text { Nível III - } 20 \text { a } 49 \% \text { = qualidade pouco satisfatória; } \\
& \text { Nível IV - } 0 \text { a } 19 \% \text { = qualidade Insatisfatória. }
\end{aligned}
$$


NEVES, E.P. e colaboradores - Tentativa de verificação da sensibilidade de um instrumento para avaliar aspectos biológicas dos cuidados de enfermagem. Rev. Bras. Enf.; DF, 29 : 42-52, 1976.

\subsection{Fórmula para o cálculo dos niveis:}

Cada observadora registrou no instrumento as respostas correspondentes. $O$ resultado dos pontos atribuídos foi calculado pela seguinte fórmula:

$$
\begin{aligned}
x= & \frac{a}{d-c} \times 100 \text { no qual } \begin{array}{l}
a=s \\
b=n(\text { sim) } \\
b=0)
\end{array} \\
c= & 0 \text { (zero) = não se aplica; } \\
d= & \text { número de ítens do instrumento; } \\
x= & \text { nível dos cudidados de enferma- } \\
& \text { gem }(\%)
\end{aligned}
$$

5. ESPECIFICAÇAO DO GRAU DE ACEITABILIDADE NA OBTENÇAO DOS OBJETIVOS:

Não constituiu preocupação para as pesquisadoras determinar até que ponto os resultados (objetivos) das intervenções seriam ou não aceitáveis. $O$ critério foi o de considerar aceitável qualquer cuidado obtido através das fontes de informação.

\section{ESPECIFICAÇAO DAS FONTES DE INFORMAÇAO:}

- Observação direta dos pacientes

- Anotações de enfermagem nos prontuários

- Informações diretas dos próprios pacientes

- Informações de outros pacientes.

\section{ELABORAÇAO E TESTAGEM DOS INSTRUMENTOS:}

\subsection{Elaboração:}

Como foi citado anteriormente, consultou-se a bibliografia disponível e utilizou-se a experiência profissional das avaliadoras. Por não constar o anexo referido na cópia xerox ainda não publi- cada da pesquisa de Brownie et Collins e devido ao interesse em conhecer o dito instrumento, foi solicitado através da Biblioteca Central da U.F.R.J. à New York Academy of Medicine Library, tendo sido o mesmo entregue às pesquisadoras, quando estas estavam já na fase de elaboração do presente relatório.

\subsection{Primeiro teste:}

As seis (6) avaliadoras foram divididas em dois (2) grupos de três (3). Um grupo avaliou os pacientes do Hospital I e II, outro do Hospital III. Os pacientes foram observados simultaneamente por 3 avaliadoras, uma vez no período matutino (9 às 12 hs.) e outra no noturno (20 às 23 hs.), sendo que estas, durante a observação, não podiam comunicar-se entre si.

\subsection{Segundo teste:}

Para esta etapa, foi reformulado o instrumento, sendo excluídos os itens que não alcançaram o mínimo de $70 \%$ de concordância e outros cuja redação carecia de revisão por sugestão das enfermeiras dos hospitais em estudo e das próprias pesquisadoras.

Dez dias após o primeiro teste, foram colhidos os dados para o $2 .^{\circ}$ teste. Neste, a amostra, que se pretendia fosse a mesma, ficou reduzida a 5 pacientes, devido as seguintes ocorrências: No hospital I, todos os pacientes estavam com o período ultrapassado. No hospital II apenas 1 paciente pode ser observado, pois dos demais, 1 estava com o período ultrapassado, 1 teve alta e 1 faleceu. No hospital III, 1 paciente foi transferido e 3 haviam ultrapassado o período estabelecido para o estudo.

Os critérios e a metodologia de avaliação do $2 .^{\circ}$ teste foram os mesmos utilizados no 1.0 teste. 
NEVES, E.P. e colaboradores - Tentativa de verificação da sensibilidade de um instrumento para avaliar aspectos biológicos dos cuidados de enfermagem. Rev. Bras. Enf.; DF, 29 : 42-52, 1976.

\section{RESULTADOS E DISCUSSAO:}

O nível dos cuidados de enfermagen: (x) atribuido as observaçōes de cada uma das avaliadoras fol obtido pela soma das respostas positivas (a) dividida pela diferença entre a soma das respostas negativas (d) e a soma das respostas nulas (c), multiplicando por 100.
Para verificar a sensibilidade do instrumento do 1.0 teste, foram calculados a média, o desvio padrão e o coeficiente de variação (C.V.) de cada avaliadora, a partir das percentagens obtidas nas observaçōes dos pacientes excluindo-se os itens que não obtiveram $\mathbf{7 0 \%}$ de concordância. Verificou-se homogeneidade entre as avaliadoras, conforme demonstra a tabela abaixo:

TABELA I: NIVEIS DE CUIDADOS DE ENFERMAGEM DOS PACIENTES DO PRIMEIRO TESTE, EM PERCENTAGEM (\%), RIO DE JANEIRO, 1975.

\begin{tabular}{|c|c|c|c|c|}
\hline \multirow{2}{*}{ Avaliadoras } & Pacientes & \multicolumn{3}{|c|}{ Avaliadoras } \\
\hline & $\begin{array}{llllllllllllllll}1 & 2 & 3 & 4 & 5 & 6 & 7 & 8 & 9 & 10 & 11 & 12 & 13 & 14 & 15 & 16\end{array}$ & & $\mathbf{S}$ & C.V \\
\hline $\mathbf{A}$ & $4872685478557881-------\cdots$ & 68 & 11 & 16 \\
\hline B & $5470715979587876--------$ & 68 & 8 & 12 \\
\hline $\mathbf{C}$ & $6668706366557574--------$ & 68 & 9 & 12 \\
\hline $\mathbf{D}$ & $--ー-ー-ー-5453575455625050$ & 54 & 7 & 13 \\
\hline $\mathbf{E}$ & $-ー-ー-ー-ー 5045566652634348$ & 54 & 7 & 13 \\
\hline $\mathbf{F}$ & - - - - - - 3642464249564648 & 47 & 7 & 15 \\
\hline $\begin{array}{l}\text { Média dos } \\
\text { Pacientes }\end{array}$ & 56707050745677474747535452604649 & 一 & 一 & 一 \\
\hline
\end{tabular}

FONTE: Observaçōes dos pacientes com A.V.C. nos hospitais II e III.

No 2.0 teste utilizou-se o mesmo procedimento estatístico. Porém, devido à diferença do número de pacientes observados em cada grupo de avaliadoras, verificou-se a homogeneidade das avaliadoras de cada grupo separadamente (Tabela II). 
NEVas, E.P. e colaboradores - Tentativa de verificaçáo da sensibilidade de um instrumento para avaliar aspectos biológicos dos cuidados de enfermagem. Rev. Bras, Enf.; DF, 29 : 42-52, 1976.

TABELA II: NIVEIS DE CUIDADOS DE ENFERMAGEM DOS PACIENTES DO SEGUNDO TESTE, EM PEROENTAGEM. RIO DE JANEIRO, 1975.

\begin{tabular}{|c|c|c|c|c|c|c|c|c|}
\hline \multirow{2}{*}{ Avaliadoras } & \multicolumn{5}{|c|}{ Pacientes } & \multicolumn{3}{|c|}{ Avaliadoras } \\
\hline & 1 & 2 & 3 & 4 & 5 & $\bar{x}$ & $\mathbf{S}$ & C. $v$. \\
\hline $\mathbf{A}$ & 70 & 一 & 一 & - & - & 70 & 15 & 2 \\
\hline B & 70 & - & - & - & - & 70 & 15 & 2 \\
\hline $\mathbf{C}$ & 69 & - & - & - & - & 69 & 15 & 2 \\
\hline D & - & 47 & 52 & 65 & 67 & 59 & 10 & 17 \\
\hline $\mathbf{E}$ & - & 46 & 49 & 59 & 68 & 56 & 9 & 16 \\
\hline $\mathbf{F}$ & - & 35 & 46 & 42 & 64 & 48 & 10 & 20 \\
\hline $\begin{array}{l}\text { Média dos } \\
\text { Pacientes }\end{array}$ & 70 & 43 & 49 & 55 & 66 & - & - & - \\
\hline
\end{tabular}

FONTE: Observaçōes dos pacientes com A.V.C. nos hospitais I, II e III.

De modo a possibilitar a comparação entre a sensibilidade dos dois instrumentos utilizados, verificou-se o C. V. do 1.0 e do 2.0 teste, cujos resultados foram, respectivamente, $13,55 \%$ e $13,84 \%$. Daí se pode constatar que, sendo o C. V. do $10^{\circ}$ teste menor do que o do $2.0^{\circ}$ teste, comprova-se estatisticamente que o primeiro instrumento fol mais sensível para me- dir a qualidade dos cuidados de enfermagem. Todavia, a diferença entre os C. V. fol mínima $(0,29 \%)$ apesar da pequena amostra utilizada no $2 .^{\circ}$ teste. Isto faz supor que se a amostragem do 2. teste fosse mais representativa poderse-ia comprovar estatisticamente, sua primazia, sua maior.. sensibilidade, sobre o primeiro instrumento (vide gráfico). 
GRAFICO N.' I: COEFICIENTE DE VARIAÇÃO DAS AVALIADORAS NO PRIMEIRO E NO SEGUNDO TESTE. RIO DE JANEIRO, 1975.

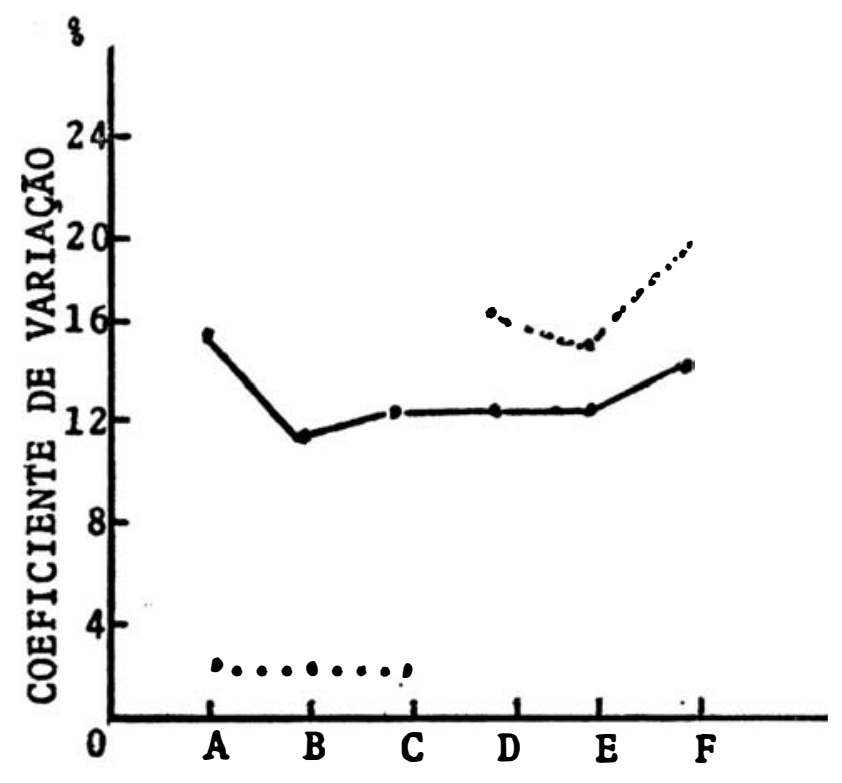

AVALIADORAS

LEGENDA:

Grupo I e II do $1^{\circ}$ teste

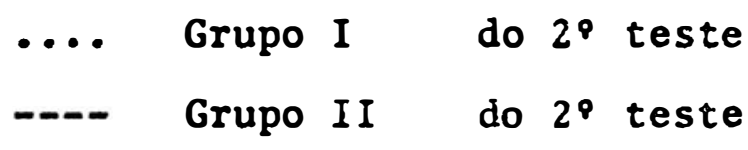

Os dados obtidos no $1 .^{\circ}$ e no $2 .^{\circ}$ teste demonstraram que o nível de cuidados de enfermagem observado na maioria dos pacientes é de moderada qualidade (Tabela III).

TABELA III: NÚMERO DE PACIENTES, POR NIVEIS DE CUIDADOS DE ENFERMAGEM, OBSERVADOS NO PRIMEIRO E SEGUNDO TESTE. RIO DE JANEIRO, 1975.

\begin{tabular}{|c|c|c|}
\hline \multirow{2}{*}{$\begin{array}{l}\text { NfVEIS DOS CUIDADOS } \\
\text { DE ENFERMAEM }\end{array}$} & \multicolumn{2}{|c|}{ NÚMERO DE PACIENTES } \\
\hline & PRIMEIRO TESTE & SEGUNDO TESTE \\
\hline ALTA QUALIDADE & - & - \\
\hline MODERADA QUALIDADE & 12 & 4 \\
\hline POUCO SATISFATORIO & 4 & 1 \\
\hline INSATISFATORIO & 一 & - \\
\hline TOTAL & 16 & 5 \\
\hline
\end{tabular}

FONTE: Pacientes com A.V.C. nos hopitais I, II e III. 
NEVES, E.P. e colaboradores - Tentativa de verificação da sensibilidade de um instrumento para avaliar aspectos biológicos dos cuidados de enfermagem. Rev. Bras. Enf.; DF, 29 : 42-52, 1976.

Para avaliar a diferença entre os níveis de cuidados de enfermagem nos períodos diurno e noturno, comprovando mais uma vez a sensibilidade do instrumento, utilizou-se o teste de hipótese de diferença entre duas proposiçōes pelo /t/ modular de Student. Fol aceita a hipótese nula (Ho) de que não há diferença significativa entre o nível de cuidado de enfermagem observado no período diurno e o do período noturno. Este procedimento não fol aplicado no segundo teste em virtude da amostragem ser insuficiente.

A comparação entre as resultados do presente estudo e os obtidos por Brownie et Collins (1974) deixou de ser feita por terem sido utilizados métodos diferentes.

Acredita-se que o método de observação por três avaliadoras simultaneamente, facilitou o controle das variáveis e a redução dos erros de medida.

Para um estudo mais completo, poderse-ia colher dados que possibilitassem correlacionar o nível de dependência física dos pacientes com o nível dos cuidados de enfermagem por eles recebidos. Pensa-se na possibilidade de existir uma correlação inversa entre as variáveis, pois as pacientes que apresentaram níveis de cuidados mais elevados foram os que menos dependiam da enfermagem.

\section{CONCLUSAO:}

Apesar de comprovada a sensibilidade do instrumento seria interessante que 0 mesmo fosse testado por um grupo constituido por uma enfermeira da própria unidade, escolhida para o estudo, uma de outra unidade e uma terceira não pertencente ao quadro de pessoal do hospital, utilizando uma amostragem mais ampla de pacientes selecionados por níveis de dependência física.

\section{RECOMENDAÇAO:}

Considerando a inexistência no pais de instrumentos para avaliar e garantir qualidade da assistência de enfermagem, recomenda-se:

AS (AOS) ENFERMEIRAS (OS) E CHEFES DOS SERVIÇOS DE ENFERMAGEM:

- que reflitam sobre a importância do assunto e procurem desenvolver pesquisas neste campo.

\section{RESUMO:}

Estudo de verificação da sensibilidade de um instrumento para medir a qualidade dos cuidados de enfermagem relacionados com as necessidades fisiológicas, em 16 pacientes portadores de Acidente Vascular Cerebral, após 72 horas de internação na clínica, até à quarta semana. $O$ instrumento elaborado, fol testado e reformulado para o segundo teste, sendo utilizado o método da observação simultânea por dois grupos de três enfermeiras, alunas do Curso de Mestrado da Escola de Enfermagem Ana Néri da UFRJ, não pertencentes aos três hospitais oficiais em estudo. Ficou comprovada a sensibilidade do instrumento e sugerido que o mesmo fosse testado por um grupo de enfermeiras, com outras características, ampliando-se a amostragem e selecionando os pacientes por níveis de dependência física. 
NEVES, E.P. e colaboradores - Tentativa de verificaçāo da sensibilidade de um instrumento para avaliar aspectos biológicos dos cuídados de enfermagem. Rev. Bras. Enf.; DF, 29 : 42-52, 1976.

\section{BIBLIOGRAFIA:}

\section{I - CITADA NO TEXTO:}

1. ABDELLAH, F. G. - Critérios de Avaliaçāo em enfermagem. Nursing Research 10 (1) : 21-26, winter 1961. Traduçāo de Circe de Mello Ribeiro In: Revista Brasileira de Enfermagem (1 e 2) : 17-31, jan. fev. 1973.

2. BERG, Helen V. - Nursing audit and outcome criteria. Nursing Clinics of North America 9 (2): 331-335, June, 1974.

3. BLOCH, Doris - Evaluation of Nursing Care in terms of process and outcome: inssues in Research and Quality Assurance. Nursing Research. 24 (4) : 256-263. Jul. Aug. 1975.

4. BROWNIE, Joan F. and COLLINS, Patricia H. - A tool to measure nursing care of patients with a cerebrovascular accident in aciente in aciente phase of illness. Journal N. Y. S. N. A., 5 (1): 20-25, March, 1974.

5. DU GAZ, Beverly Witter - Valoracion de la necessidad de la enfermeria in: Tratado de Enfermeria Practica de Kozier, 2. Ed. Interamericana, México, 1974.

6. KAPLAN, Abraham - Medidas, in: $A$ condutc na pesquisa, $1 .^{\mathrm{a}}$ ed., Editora Herder, S. Paulo, 1969, págs. 203-208.

7. TAYLOR, Joyce W. Measuring the outcomes of Nursing Care Nursing Clinics of North America. 9 (2): 337-348, June 1974.

8. ZIMMLER, Marie J. Quality assurance of outcomes of patient care, Nursing Clinics of North America 9 (2): 305-315, Jun. 1974.

9. ZIMMER, Marie J. et alii - Guidelines for development of outcome criteria. Nursing Clinics of North America 9 (2): 317321, Jun., 1974.
II - CONSULTADA:

1. ABDELLAH, Faye and LEVINE, Eugene - Better patient care through nursing research ed. Mac-Millan, New York, 1965.

2. CERVO, Amado Luiz e BERVIAN, Pedro Alcino - Metodologia Cientifica, 1.8 ed. Mc GrawHill, S. Paulo, 1975.

3. FELTON, Geraldine - Increasing the quality of nursing care by introducing the concep of primary nursing: a model project. Nursing research 24 (1): 27-32, Jan. Feb. 1975.

4. FERRELRA, Aurélío Buarque de Holanda - Novo Dicionário $d a$ Lingua Portuguesa, 1. ${ }^{\mathrm{a}}$ Ed. Nova Fronteira, Rio de Janeiro, 1965.

5. GOODE, Margaret - The patient with a cerebral vascular accident. Nursing outlook 14 (3): 60-62, March, 1966.

6. GOODE, Willian J. e HATT, Paul K. - Métodos em Pesquisa Social. 4." Ed. Companhia Editora Nacional, 1973.

7. HANSON, Robert L. - Research in Nursing Service. Nursing outloock 19 (8): 520-523, Aug., 1971.

8. HENDERSON, Virginia - Principios Básicos sobre cuidados de Enfermagem. ABEn e F.SESP Rio de Janeiro, 1962.

9. NICHOLLS, Marion E. - Quality Control in Patient, American Journal of nursing 74 (3): 456-459, Mar. 1974.

10. RIBEIRO, Circe le Melo - Auditoria no Serviço de Enfermagem, Revista Brasileira de Enfermagem (4): 91-102, Jul-Set. 1972.

11. RUBIN, Charlene F. RINALDI, Leena A. and DIETZ, Ruth Nursing evaluating nursing, American Journal of Nursing 72 (5): 916-921, May, 1972. 
NEVES, E.P. e colaboradores - Tentativa de verificação da sensibilidade de um instrumento para avaliar aspectos biológicos dos cuidados de enfermagem. Rev. Bras. En?.; DF, 29 : 42-52, 1976.

12. SELLTIZ et alii - Métodos de Pesquisa nas Relaçōes Sociais, 5. Ed, Editora Pedagógica e Universitária, S. Paulo, 1975.

13. SMITR, Doroty, GERMAIN, Carol P. Hauley e GIPS, Claudia D. Acidente Vascular Cerebral, in: Enfermeria Medico-quirúrgica, 3." Ed. Interamericana, México, 1973. Págs. 1150-1164.
14. WANDFLT, Mabel - Guide for the Beginning Researcher 1st Ed., Meredith corporation, New York, 1970.

15. WOLF', Harvey - Can nonnurses make qualitative observátions of nursing care? Nursing outlook 13 (2): 52-53 Feb., 1965. 
Instrumento para mensuração dos cuidados de enfermagem prestados aos pacientes neurológicos - A. V. C., relacionados com as necessidades biológicas, após 72 horas de instalação da doença até a quarta semana.

Código do Hospital: Unidade:

Nome do paciente: Leito: Idade:

Sexo:

Visita:
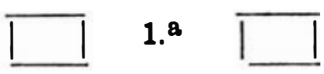

2.8

Periodo:

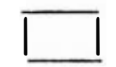

Diurno

Noturno

Data da admissão:

Hora da admissão:

Data da avaliação:

Hora:

Nome da avaliadora:

\section{SUMARIO DAS CONDIÇÓES DO PACIENTE}

1. nível de consciência:

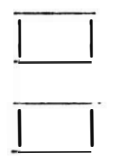

consciente

$\square$ inconsciente confuso

2. condições de comunicação:

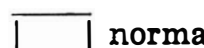
normal

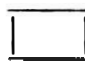
afásico

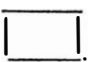
disártrico

3. condições de deambulação:

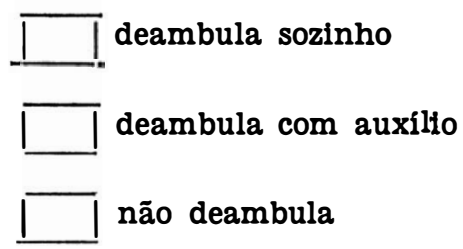

4. condições de mobllização no leito: mobiliza sozinho $\square$ mobiliza com auxílio

5. presença de sondas:

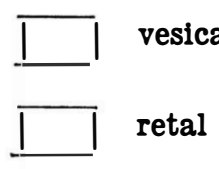

6. presença de cânulas:

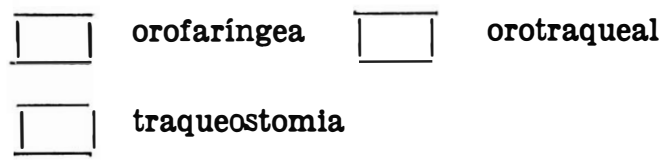




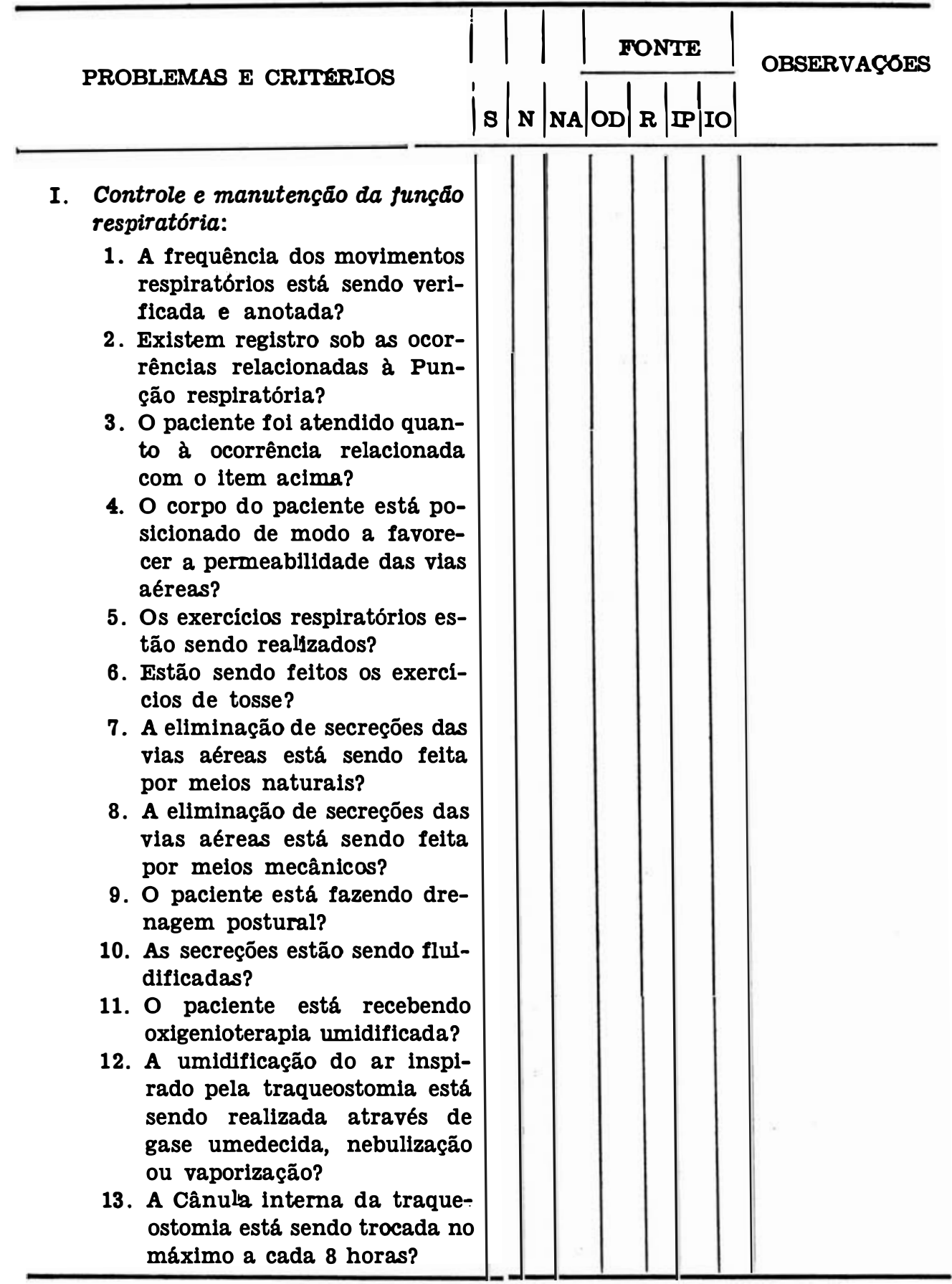

Legend a: $\mathbf{S}=$ Sim, cuidado prestado

$$
\begin{aligned}
\mathbf{N}= & \text { Não, " não prestado } \\
\mathbf{N} . \mathbf{A}= & \text { Não se aplica, o paciente } \\
& \text { não necessita }
\end{aligned}
$$

OD. = Observação direta do paciente

R. = Verificação nos registros

I.P. = Informaçōes do próprio paciente

I.O. = Informaçōes de outro(s) paciente ( $\mathrm{s}$ ) 


\section{S $|\mathbf{N}| \mathrm{NA}|\mathrm{OD}| \mathbf{R}|\mathrm{IP}| \mathrm{IO}$}

II. Controle e manutenção das funçôes de alimentação e hidratação pelas vias naturais e/ou artificiais:

1. O tipo de dieta é adequado às condições do paciente?

2. Existem registros sobre ingestão e aceitação da dieta?

3. Existem registros sobre a ingestão e aceitação de líquidos?

4. A dieta está balanceada em relação ao horário?

5. O paciente é auxiliado ao se alimentar?

6. O paciente é auxiliado na ingestão de líquidos?

7. A sonda nasogástrica é mantida limpa?

8. O paciente é colocado em posição de Fawler quando alimentado por SNG ou por via natural?

III. Controle e manutenção das funçōes de eliminação:

1. Existem registros sobre a frequência das eliminações de urina?

2. Existem registros sobre a quantidade de urina eliminada?

3. Existem registros sobre as características da urina (corodor-turvação)?

4. Existem registros sobre a frequência das eliminações de fezes?

5. Existem registros sobre as características das fezes (cor, consistência, odor, elementos anormais)?

6. Existem registros sobre quantidade e característica dos vômitos? 
7. Existem registros sobre as características das secreçōes?

8. Existem registros sobre a quantidade de secreção eliminada?

9. O meato urinário dos pacientes sondados está limpo?

10. O meato urinário dos pacientes sondados foi pincelado com mercúrio cromo?

11. Os coletores ou frascos estão limpos?

12. As conexões e intermediários estão limpos?

13. Existem registros que permitam controlar o tempo de permanência da sonda vesical?

14. A sonda é pinçada durante algumas horas, para reeducação vesical dos pacientes conscientes?

IV. Controle e manutenção das funçōes reguladoras:

1. O T.P.R. é verificado de acordo com as condiçōes do paciente?

2. A tensão arterial é verificada de acordo com as condiçōes do paciente?

3. Existem registros sobre medidas tomadas para atender às alteraçōes das funçōes reguladoras? (TPR - TA; sudorese, balanço hídrico, cianose)

4. O ambiente favorece aquecimento, ventilação e lluminação de acordo com as necessidades do paciente?

5. Existem registros sobre a quantidade de liquidos ingeridos por via oral ou SNG? 
6. Existem registros sobre a natureza e quantidade dos líquidos administrados por via endovenosa?

7. Existem registros sobre o balanço hídrico das 24 horas e nos pacientes graves?

V. Promoção e manutenção do cuidado corporal, conforto e integridade fisica:

1. A cavidade bucal está limpa?

2. Os lábios ressequidos estão lubrificados?

3. Os olhos estão sendo umidificados limpos e/ou protegidos?

4. O corpo está limpo?

5. Os cabelos estão limpos?

6. Os cabelos estão penteados?

7. As unhas estão limpas?

8. Está recebendo massagens de conforto?

9. A SNG está fixada de modo a evitar traumatismos?

10. As contençōes estão sendo aplicadas de modo a evitar traumatismos?

11. A Sonda vesical está fixada de modo a evitar traumatismos?

12. A cânula de traqueostomia está fixada de modo a evitar traumatismos?

13. As áreas contíguas as saliências ósseas estão protegidas de modo a evitar escaras?

14. Os lençóis dos pacientes acamados estão limpos, secos e esticados?

15. O paciente apresenta sinais de providências tomadas para aliviar lesōes causadas por esparadrapos? 


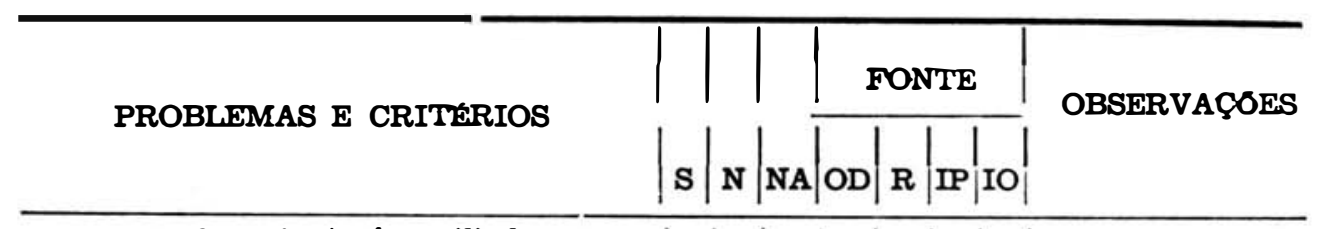

16. O paciente é auxiliado a mudar de decúbito cada 2 ou 3 horas?

17. O paciente está posicionado de modo a manter a postura e a proporcionar conforto?

18. A unidade do paciente está limpa?

19. A comadre está limpa?

20. A comadre está acessível (se o paciente puder usá-la sozinho)?

21. O papagaio está limpo?

22. O papagaio está acessível?

VI. Promoção e manutenção da segurança física?

1. Há identificação no leito do paciente? (n. $\left.{ }^{\circ}\right)$

2. $A(s) \operatorname{grade}(s)$ do leito estāo levantadas?

3. A campaínha está acessível?

4. A campainha funciona?

5. O paciente está recebendo cuidados que nāo ameaçam sua segurança? (só computar se observado)

6. As contençōes estāo aplicadas de modo a evitar que o próprio paciente ameace sua segurança?

7. A mesa de cabeceira está livre de objetos que possam ameaçar sua segurança?

8. O paciente está livre de lesões cutâneas provocadas por agentes físicos e/ou químicos?

9. Os curativos estāo limpos?

10. Os frascos de soluções parenterais que o paciente está recebendo estāo identificados? (nome do paciente, leito, conteúdo, doses, n.o de frasco, hora, n.o de gotas/minuto).

11. A SNG é testada antes de ser administrada qualquer substância ao paciente? 
I $\mathbf{S}|\mathbf{N}| \mathbf{N A}$ OD $\mathbf{R}$ IP $\mid \mathrm{IO}$

VII. Controle e manutenção das funçôes sensoriais e motoras:

1. Existem registros que indiquem controle sobre o nível de consciência?

2. Existem registros sobre os reflexos?

3. Existem registros sobre a tonicidade muscular? (flacidez, hipertomia, contraturas).

4. Existem registros sobre medidas tomadas para o alívio da dor?

5. Os segmentos corporais estão posicionados de modo a evitar deformidades?

6. Foram tomadas medídas com relação a deambulação?

7. O paciente tem sido auxillado a sair do leito e a sentar-se na cadeira?

8. Foram tomadas medidas com relação aos exercícios passivos e/ou ativas no leito?

VIII. Promoção e manutenção das condiçōes favoráveis à terapêutica:

1. Existem registros das medicaçōes administradas (nome dose - via - horário - dia rubrica)?

2. Existem registros de reações apresentadas pelos pacientes, com relação a terapêtica?

3. Há sinais de aplicação de medicamentos parenterais, em rodízio, nas diversas regiōes recomendadas? (excluir os membros paralíticos)

4. Há sinais de providências para aliviar alterações nos locais de aplicação dos medicamentos parenterais?

5. O gotejamento das soluções endovenosas está de acordo com o recomendado? 